\title{
Exoscopic visualisation with VITOM@ 3D in cochlear implantation: preliminary results
}

\author{
Yury Rusetsky ${ }^{1}$, Nataliya Chuchueva ${ }^{1}$, Irina Meytel $^{2}$, Uliana Malyavina ${ }^{2}$, and Seitmurat \\ Farikov $^{1}$ \\ ${ }^{1}$ Central State Medical Academy \\ ${ }^{2}$ FSAI National Medical Research Center for Children's Health
}

April 25, 2021

\begin{abstract}
Key points - 3D exoscopy is an emerging visualisation technique designed to improve ergonomics and image quality during

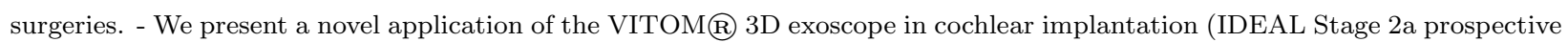
case series). - The system enabled high-quality visualisation during both posterior tympanotomy and electrode insertion. - Both the chief surgeon and the staff members rated the ergonomics of the system highly. - 3D exoscopy is a useful alternative to conventional microscopy, but the two techniques remain to be directly compared in larger studies.
\end{abstract}

\section{Key points}

- 3D exoscopy is an emerging visualisation technique designed to improve ergonomics and image quality during surgeries.

- We present a novel application of the VITOM $®$ 3D exoscope in cochlear implantation (IDEAL Stage 2a prospective case series).

- The system enabled high-quality visualisation during both posterior tympanotomy and electrode insertion.

- Both the chief surgeon and the staff members rated the ergonomics of the system highly.

- 3D exoscopy is a useful alternative to conventional microscopy, but the two techniques remain to be directly compared in larger studies.

\section{Introduction}

The development of three-dimensional (3D) endoscopy and its exoscopic application opened up new avenues for the surgery of anatomic microstructures ${ }^{1}$. An exoscopic camera has a longer focal distance than a microscope, which allows surgeons to assume a more ergonomic position during surgery. 3D and 2D monitors also provide a better view of the surgical site to assistants and residents.

3D exoscopy has already been applied in otorhinolaryngology with positive outcomes. Ahmad et al. presented the largest case series of free flap dissection and microvascular anastomosis performed under 3D vision ${ }^{2}$. Authors reported both superior lighting and better ergonomics provided by a $3 \mathrm{D}$ exoscope compared to a conventional microscope. Several groups of authors reported positive impressions of 3D-assisted lateral skull base surgeries ${ }^{3,4}$.

Cochlear implantation is a promising area for application of 3D visual control. Firstly, this intervention has a clear sequence of mandatory surgical steps, and, secondly, it requires high magnification and efficient spatial orientation in the deep regions of the middle ear. The goal of this study was to assess the performance of the VITOM@ 3D exoscopic system during cochlear implantation and to refine the technique if necessary. 


\section{Methods}

\subsection{Subjects}

Based on the established criteria for cochlear implantation, subjects were selected for a prospective case series between 1 September and 1 October 2020 at the National Medical Research Centre for Children's Health. All subjects signed an informed consent form. The study was approved by a local ethics committee and was conducted in accordance with the Declaration of Helsinki.

\subsection{Surgical procedure}

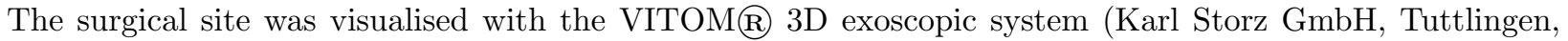
Germany). The system consisted of a 3D high-resolution (4K) camera, a joystick, a processing module (Image 1S+ D3-LINK), and a 3D monitor (HD or 4K). The camera allowed $8-30 \mathrm{x}$ magnification at a focal distance of $20-50 \mathrm{~cm}$. Polarised glasses were used to enable 3D vision.

The arrangement of the equipment relative to the operation table and the staff is shown in Figures 1-2. The 3D monitor was placed at the eye level of the chief surgeon and the scrub nurse. The audience consisting of residents followed the procedure on the same screen, while the assistant used the $2 \mathrm{D}$ monitor. The $3 \mathrm{D}$ camera was placed $30-35 \mathrm{~cm}$ away from the surgical site (recommended minimum distance is $20 \mathrm{~cm}$ ). The joystick was nearby, within reach of the chief surgeon. Between surgeries, the camera and the joystick arms were stowed safely by moving them away from the operation table to the level of the tripod mount.

Prior to the surgeries, all staff members were instructed by an expert technician on how to use the VITOM@ 3D system. The technician also provided support during the first surgeries. The first author was the chief surgeon during all surgeries. Standard cochlear implantation procedure was implemented. The initial external steps of the intervention (skin and soft tissue incision, mastoidectomy, preparation of an implant bone bed) were performed under conventional surgical lighting, without any magnification. Posterior tympanotomy, electrode insertion, and the stapedius reflex check were performed using the 3D monitor of the VITOM@ $3 \mathrm{D}$ system with appropriate $(8-30 \mathrm{x})$ magnification. A ready-to-use microscope was also available. Patients were implanted with the Nucleus system from Cochlear Limited $(\mathrm{n}=18)$, SONATA from MED-EL $(\mathrm{n}=7)$, and HiRes90K from Advanced Bionics $(\mathrm{n}=3)$.

\subsection{Outcome measures}

Intra- and post-operative complications were recorded. The 3D image quality was assessed in terms of the spatial orientation and visibility of the middle ear anatomy. More specifically, we looked at three parameters: stereoscopy, lighting, and focusing during posterior tympanotomy and electrode insertion. Surgeon comfort was assessed in terms of physical discomfort and eye strain. Staff comfort was assessed in terms of equipment ergonomics and eye strain. All parameters were rated on a 5-point Likert scale, where 5 meant "very good", 4 - "good", 3 - "neutral", 2 - "bad", and 1 - "very bad". The assessment was performed by the chief surgeon immediately after each surgery. The mode of the answers by the scrub nurse, circulating nurse, and three residents was used as a measure of staff comfort. The percentage of surgeries falling under each Likert category was calculated.

\subsection{Reporting Guideline}

The manuscript was prepared according to the IDEAL Stage 2a guideline designed for reporting the evaluation and development of innovative surgical techniques.

\section{Results}

28 patients (16 males, 12 females) underwent cochlear implantation. Their mean age was 4.5 years (range: 1.5 - 9.4). There were no technical difficulties that forced the surgeon to switch to the microscope. This means both planned surgical steps were performed solely with VITOM( 3 D. Initially, the tripods were at the surgeon's side, but this arrangement restricted the surgeon's hand movements slightly; therefore, the tripods were moved to the other side after the second surgery. Mean time was 7.4 min (range: $6.0-9.0$ 
$\min$ ) for equipment preparation prior to the surgery. Mean time was 9.9 min (range: 4.6 - 24.0) for posterior tympanotomy and 3.0 min (range: 1.0 - 19.0) for electrode insertion. The time outliers in 2 cases were due to anatomical abnormalities. No patients experienced intra- or post-operative complications. In all cases, responses of the auditory nerve were obtained after the first insertion of the electrode.

Individual scores are presented in chronological order in Table 1. The frequency distributions of the scores are presented in Table 2. All parameters of the VITOM@ 3D system use were rated between "neutral" and "very good". Staff comfort was rated "very good" in $82.1 \%$. Surgeon comfort was rated "very good" in $75 \%$ of cases. Lighting and focusing during posterior tympanotomy was better than during electrode insertion. Overall, the scores improved throughout the surgeries.

\section{Discussion}

In our case series, the VITOM@ 3D system demonstrated satisfactory performance during cochlear implantation. The outcome measures gradually improved and reached stability for both posterior tympanotomy and electrode insertion. There was no need to switch to the microscope. The chief surgeon rated the quality of surgical images very highly: all visual parameters were "good" or "very good" in over $80 \%$ of cases. Residents followed the procedure on a 3D monitor with preserved depth perception and excellent resolution, which facilitated the educational process. Our findings are in agreement with other studies reporting positive results of 3D-exoscopic visualisation in otosurgery ${ }^{3,4}$. Rubini et al reported high image quality produced by the new tool in a case series of lateral skull base procedures including one cochlear implantation ${ }^{3}$. They also noted, however, that the lighting of the deep regions was sometimes suboptimal. Also in our study, the lighting during electrode insertion was rated "very good" in only $50 \%$ of cases.

3D technologies are also expected to allow the surgeon to assume a more ergonomic position thanks to the adjustable monitor height. Compact camera and joystick are designed not to restrict hand movements but still be within surgeon's reach. Our findings confirmed that: the chief surgeon rated his comfort during the surgery as "very good" in $75 \%$ of cases. This result is in agreement with other studies, which also reported more ergonomic positions when a $3 \mathrm{D}$ exoscope was $\mathrm{used}^{2-4}$. A pedal could be a more convenient image controller than a joystick, because it would allow surgeons to keep both hands at the operating site at all times.

The entire VITOM( 3 D system is relatively small and provides better ergonomics in the surgery room than a microscope. It allows circulating nurse and other staff to move around easily. Unlike a microscope, VITOM@ 3D did not have to be moved away entirely between consecutive surgeries if they were performed on the same side. Only the camera and the joystick were stowed away safely beside the operating table by turning the tripods.

The absence of a second 3D monitor was a disadvantage in our setting. 3D visualisation is obtained when the monitor is positioned directly in front of the person. During cochlear implantation, the chief surgeon and the assistant were at a 90-degree angle to each other. Therefore, the assistant could not directly observe the primary monitor and would have benefited from another 3D monitor. Limited sample size did not allow us to compare the time of electrode insertion between different implant types. This could be a useful comparison in future studies.

In the first 3D exoscope-assisted cochlear implantation case series, we demonstrated that the VITOM@ 3D system could be used for different cochlear implant types and both implantation sides. Based on our preliminary findings, 3D exoscopy may perform as well as conventional microscopy in terms of time, visualisation, lighting, instrument manipulation, and the range of possible intervention stages. The new technology may outperform microscopy in terms of manoeuvrability, ergonomics, comfort, and educational value. Taken together, these characteristics make the VITOM@ 3D system a promising surgical tool. Larger case series and studies with group comparisons are required to establish guidelines for VITOM@3D-assisted cochlear implantation.

\section{References}


1. Nishiyama K. From exoscope into the next generation. J Korean Neurosurg Soc. 2017;60(3):289-293. doi:10.3340/jkns.2017.0202.003

2. Ahmad FI, Mericli AF, DeFazio MV., et al. Application of the ORBEYE three-dimensional exoscope for microsurgical procedures. Microsurgery. 2020;40(4):468-472. doi:10.1002/micr.30547

3. Rubini A, Di Gioia S, Marchioni D. 3D exoscopic surgery of lateral skull base. Eur Arch Oto-RhinoLaryngology. 2020;277(3):687-694. doi:10.1007/s00405-019-05736-7

4. Gordon SA, Deep NL, Jethanamest D. Exoscope and Personal Protective Equipment Use for Otologic Surgery in the Era of COVID-19. Otolaryngol - Head Neck Surg (United States). 2020;163(1):179-181. doi:10.1177/0194599820928975

5. De Virgilio A, Iocca O, Di Maio P, et al. Free flap microvascular anastomosis in head and neck reconstruction using a 4K three-dimensional exoscope system (VITOM 3D). Int J Oral Maxillofac Surg. 2020;49(9):11691173. doi:10.1016/j.ijom.2020.01.022

\section{Tables and Figures}

1. Figure 1. Operating room layout (for a surgery on the left ear).

2. Figure 2. Equipment arrangement during VITOM@ 3D-assisted cochlear implantation.

3. Table 1. Individual scores for each surgery

4. Table 2. Questionnaire results

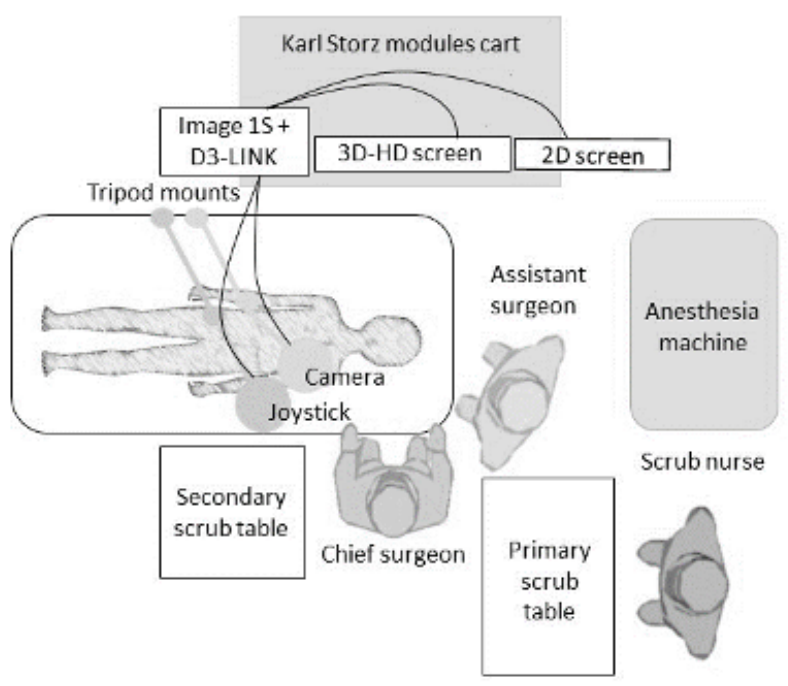




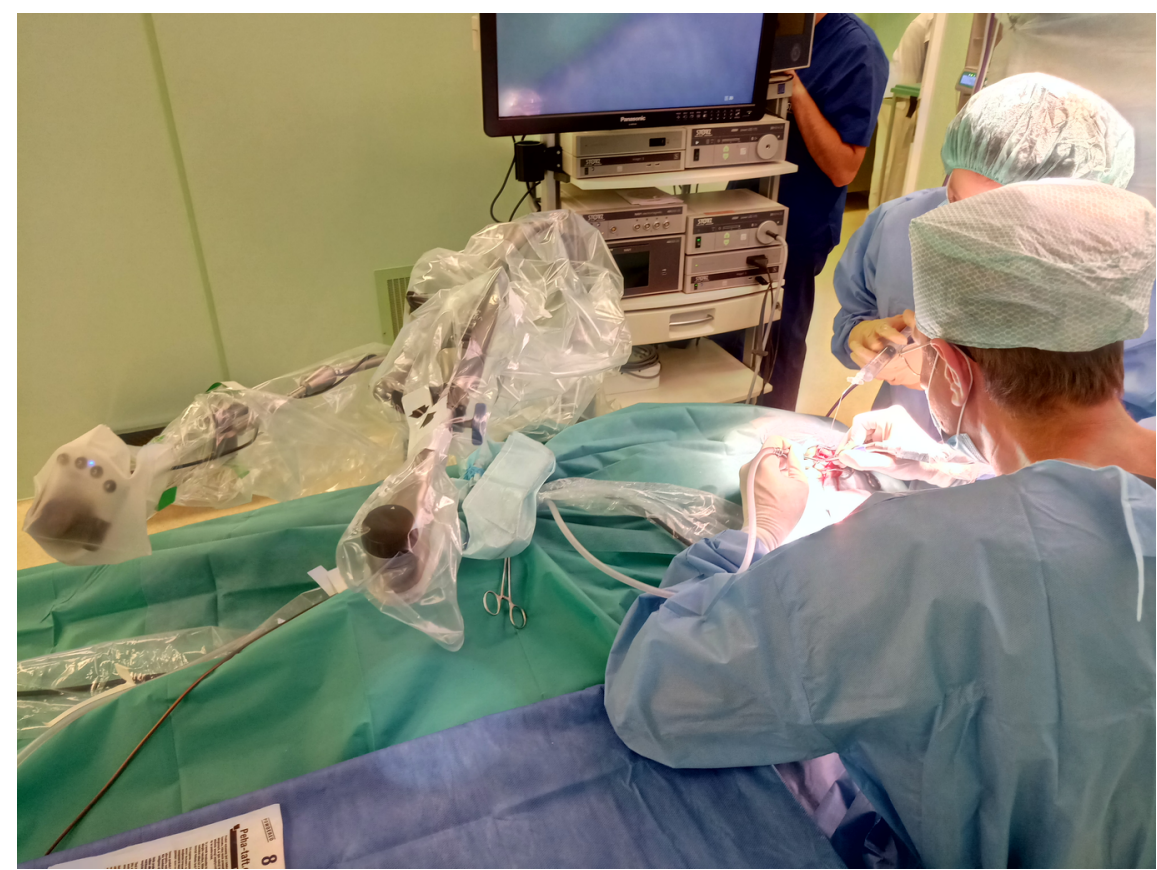

\section{Hosted file}

Table 1.pdf available at https://authorea.com/users/409934/articles/519461-exoscopicvisualisation-with-vitom-3d-in-cochlear-implantation-preliminary-results

\section{Hosted file}

Table 2.pdf available at https://authorea.com/users/409934/articles/519461-exoscopicvisualisation-with-vitom-3d-in-cochlear-implantation-preliminary-results 